\title{
Genotyping and Antifungal Susceptibility Profile of Sequential Candida albicans Isolated from the Oral Cavity of HIV-Infected Individuals
}

Daniela Vanessa Moris², 2, 3 , Joyce Marinho de Souza ${ }^{3}$, Márcia Souza Carvalho Melhem4, Marilena dos Anjos Martins $^{4}$, Caroline Lucio Moreira ${ }^{3}$, Lenice Rosario de Souza ${ }^{1}$, Gioconda San-Blas ${ }^{5}$, Maria Walderez Szeszs ${ }^{4}$, Lidia Raquel Carvalho $^{6}$, Marcus Vinícius Pimenta-Rodrigues ${ }^{2,3}$ and Rinaldo Poncio Mendes ${ }^{1}$

1. Tropical Diseases Area_Faculdade de Medicina de Botucatu, Universidade Estadual Paulista "Júlio de Mesquita Filho"-UNESP, Sao Paulo Botucatu 18600-000, Brazil

2. Masters in Health Science — Universidade do Oeste Paulista_-UNOESTE, Sao Paulo Presidente Prudente 19067-175, Brazil

3. Department of Biomedical Sciences_-Universidade do Oeste Paulista_-UNOESTE, Presidente Prudente 19050-920, Brazil

4. Instituto Adolfo Lutz, Sao Paulo 01246-000, Brazil

5. Venezuelan Institute for Scientific Research, Center for Microbiology and Cell Biology, Caracas 11 01204, Venezuela

6. Biostatistics Department, Instituto de Biociências, Universidade Estadual Paulista “Júlio de Mesquita Filho”—UNESP, Sao

Paulo Botucatu 18600-000, Brazil

\begin{abstract}
Objective: The study aimed to evaluate the genotypic profiles of C. albicans (Candida albicans) sequentially isolated throughout the course of HIV infections, and to determine its MIC (minimal inhibitory concentrations) to AMB (amphotericin B), FLC (fluconazole), KTC (ketoconazole), and ITC (itraconazole). Design: samples were collected from the oral cavity of HIV-positive individuals during 4 years, with a sterilized swab. MIC was performed by using the microdilution method AFST/EUCAST. The genetic similarities within and between sequential clones of $C$. albicans were assessed by DNA fingerprinting using the random amplification of polymorphic DNA technique. Results: A total of 142 oral samples were isolated from 59 HIV-infected individuals who attempted up to five visits each, with or without symptoms of oropharyngeal candidiasis. Profile analysis revealed that yeasts isolated over sequential visits from symptomatic or asymptomatic individuals showed $78 \%$ or $87 \%$ relatedness, respectively. The degree of similarity among $C$. albicans was higher for isolates from colonization than for those from infection. Genetically identical C. albicans samples also formed connected subclusters in sequential visits. In regard to susceptibility profile, all isolates were susceptible to AMB, FLC, KTC, and ITC and maintained this pattern all along, no differences in MICs of any given antifungal compound were observed for sequential $C$. albicans isolates. Conclusions: These data suggest that genotype and susceptibility to antifungal drugs were maintained over time in sequentially isolates of $C$. albicans colonization and a diverse evolutionary genetic trend in C. albicans sequentially isolated from the oral candidiasis of HIV infected individuals.
\end{abstract}

Key words: Genotyping, Candida albicans, sequential, HIV- positive individuals, oral cavity.

\section{Introduction}

Host defense mechanisms against Candida albicans are complex. Deficiencies in both phagocytic cell functions and classical cell-mediated or humoral immune responses are linked to the increased

Corresponding author: Daniela Vanessa Moris, Ph.D., professor, research fields: mycology and microbiology. susceptibility of the host to C. albicans infection [1]. The impairment of cell-mediated immunity caused by the HIV (human immunodeficiency virus) predisposes these individuals to oral candidiasis and other opportunistic infections [2-4]. Both the prevalence of $C$. albicans colonization and the recurrence of oral candidiasis increase with the progress of HIV infection for HIV-infected individuals, oral candidiasis has been 
recognized as an early expression of their immunodeficiency, while esophageal candidiasis is considered a clinical predictor of AIDS. The repeated use of short- or long-term antifungal azoles, particularly FLC (fluconazole), to treat oropharyngeal candidiasis can lead to the development and/or selection of resistance among clinical isolates [5].

This, in turn, implies that subclones of C. albicans with variable genotypes may simultaneously colonize the oral cavity. In this case, it is difficult to determine with certainty the contribution, if any, of individual clones to infection $[6,7]$, and the presence of more than one clonal type may have important therapeutic implications [7-9]. However, few clonal variations are known for C. albicans during either recurrent episodes of oral candidiasis or asymptomatic carriage over a prolonged course of HIV infection. Recent advances in molecular techniques have generated several typing methods for the genetic assessment of strain relatedness in $C$. albicans, which in turn have facilitated detailed studies on the molecular epidemiology of this year [10]. In addition, a variety of molecular methods have indicated that $C$. albicans strains tend to be genetically similar when isolated from the same specific groups, such as HIV-positive and negative patients from geographically related locations [11].

Molecular techniques include Cp3-13-specific probe, hybridization, PFGE (pulsed-field gel electrophoresis), RAPD (random amplification of polymorphic DNA), RFLP (restriction fragment length polymorphism), AFLP (amplified fragment length polymorphism) and MLST (multilocus sequence typing) [8, 12, 13]. These techniques have helped delineate subtypes of colonizing and/or infecting C. albicans strains over sequential evaluations of HIV-infected individuals. This study aimed to evaluate the genotypic profiles of C. albicans isolated from the oral cavity over sequential clinical visits during the course of HIV infection; also, determinations of MIC (minimal inhibitory concentrations) to AMB (amphotericin B),
KTC (ketoconazole), FLC (fluconazole) and ITC (itraconazole) were carried out.

\section{Materials and Methods}

\subsection{Samples Collection and Ethical Consideration}

Samples were acquired from the oral cavity of HIV-positive individuals from June 2002 to July 2006, who attempted visits to the Special Outpatient Clinic for Infectious and Parasitic Diseases or at the Ward of Tropical Diseases of Faculdade de Medicina de Botucatu, São Paulo State University (UNESP). On each visit, samples were collected using a sterilized swab, for culture independently of the clinical signs and symptoms of oral candidiasis. Each of the 59 patients attended the clinic between two and five times during this period. The characteristics of the 59 HIV-infected individuals from which Candida spp. was isolated are shown in Tables $1 \mathrm{~A}$ and 1B. In total, 142 oral swab samples were obtained, as described below. Approval was obtained from the Research Ethics Committee, Faculdade de Medicina de Botucatu, UNESP, and from the Ethics Committee on Human Research, IAL (Instituto Adolfo Lutz). Written informed consent was obtained from every patient.

\subsection{Fungal Isolates}

The HIV-infected individuals were subjected to 142 swabs of the oral cavity. Swabs were then placed on SDA (Sabourand Dextrose Agar) plates (Difco, Detroit, Michigan) supplemented with $0.05 \%$ chloramphenicol. Plates were incubated at $30{ }^{\circ} \mathrm{C}$ for $24 \mathrm{~h}$. All yeast colonies growing on each SDA plates were suspended in physiological solution and $10 \mu \mathrm{L}$ of the suspension were used to inoculate plates of CHROMagar Candida media (CHROMagar Company, Paris, France) previous to incubation at $35^{\circ} \mathrm{C}$ for $72 \mathrm{~h}$. A presumptive identification of Candida isolates was attempted on the basis of the characteristic colors of the colonies appearing on CHROMagar Candida. When colonies from a given oral swab were morphologically homogenous, only one of them was subjected to the 
Table 1A Characteristics of the 59 human immunodeficiency virus infected individuals from whom Candida spp. were isolated in up to five different moments.

\begin{tabular}{lll}
\hline Characteristics & $\begin{array}{l}\text { Individuals } \\
\text { no. }(\%)\end{array}$ & $\begin{array}{l}\text { Swabs } \\
\text { no. }(\%)\end{array}$ \\
\hline Gender $\left(\mathrm{n}_{1}=59\right)$ & $38(64.4)$ & - \\
\hline Male & $21(35.6)$ & - \\
Female & & - \\
\hline Stage $\left(\mathrm{n}_{1}=59\right)$ & $46(78.0)$ & - \\
\hline AIDS & $13(22.0)$ & $125(88.0)$ \\
non-AIDS & & $17(12.0)$ \\
\hline Host-Candida relationship $\left(\mathrm{n}_{2}=142\right)$ & - & $18(12.7)$ \\
\hline Colonization & - & $124(87.3)$ \\
Infection & & \\
Antifungal therapy $\left(\mathrm{n}_{2}=142\right)$ & - & - \\
Yes & - & - \\
No & & \\
\hline Initial anti-retroviral therapy $\left(\mathrm{n}_{1}=59\right)$ & $46(78.0)$ & \\
\hline Yes & $13(22.0)$ & \\
No & & \\
\hline
\end{tabular}

$\mathrm{n}_{1}=$ number of individuals, $\mathrm{n}_{2}=$ number of oral swabs.

Table 1B Characteristics of the 59 human immunodeficiency virus individuals from whom Candida spp. were isolated at the moment of the first visit.

\begin{tabular}{llll}
\hline Characteristics & Patients $\mathrm{n}$. & Average & Range \\
\hline Age (years old) & 59 & 37.0 & $19.0-64.0$ \\
Initial CD ${ }^{+} \mathrm{T}$ lymphocytes $\left(\mathrm{no} . / \mathrm{mm}^{3}\right)$ & 53 & & \\
$<200$ & 23 & 93.0 & $4.0-184.0$ \\
$\geq 200$ & 30 & 336.0 & $200.0-821.0$ \\
Initial HIV viral load (copies/mL) & 50 & & - \\
Undetectable & 15 & - & $81.0-5,736$ \\
$<10,000$ & 21 & 1,119 & $11,429-2,700,000$ \\
$\geq 10,000$ & 14 & 53,757 & $1-15$ \\
Intervals among visits (months)* & 59 & 3.0 & \\
$*$ Two or more visits & & & \\
\hline
\end{tabular}

complete phenotypical identification. On the contrary, heterogeneous colonies were subcultured before a complete phenotypical identification was carried out.

To identify Candida spp., isolates were incubated in cornmeal agar (Oxoid, Ltda, Brazil) supplemented with 1\% Tween 80 (Synth, Labsynth, Brazil) to verify their morphologyby conventional methodsand in API 20C AUX system (BioMérieux Marcy I'Etoile, France) to determine their biochemical characteristics according to the manufacturer's instructions.

\subsection{DNA extraction}

Each isolate was plated on SDA and incubated at 30
${ }^{\circ} \mathrm{C}$ for 24 h. DNA was extracted by phenol/chloroform/isoamyl alcohol and precipitated with isopropanol according to Ref. [14]. DNA concentrations were determined by using a NanoDrop1000 (Thermo Fisher Scientific, USA) apparatus, as described by Green \& Sambrook (2012) [14]. For PCR and RAPD amplification, $1 \mu \mathrm{L}$ of each DNA sample (approximately $100 \mathrm{ng}$ ) was used. DNA was kept at $-20{ }^{\circ} \mathrm{C}$ until use.

2.4 Molecular Differentiation between C. albicans and Candida dubliniensis

All isolates phenotypically identified as $C$ albicans 
were subjected to molecular differentiation between $C$. albicans and C. dubliniensis by PCR (polymerase chain reaction), as previously described by Mannarelli (1998) [15] PCR profiles were compared to type strains C. albicans, ATCC (American Type Culture Collection) 76615, and C. dubliniensis, CBS (Centraalbureau voor Schimmelcultures) 9768.

\subsection{Genotyping of C. albicans Isolates}

All 140 C. albicans isolates were submitted to RAPD analysis. Amplification was carried out with the Ready-to-Go-RAPD Analysis Beads kit (GE Healthcare, United Kingdom) to the manufacturer's instructions.

RAPD beads were composed of 1.5 units of Taq DNA polymerase, $10 \mathrm{mM}$ Tris- $\mathrm{HCl} \mathrm{pH} 8.3,30 \mathrm{mM}$ $\mathrm{KCl}, 3 \mathrm{mM} \mathrm{MgCl}, 400 \mathrm{mM}$ of each dNTP, and stabilizers such as bovine serum albumin. Reactions were performed by adding each DNA template and 25 pmol of the primer to a final volume of $25 \mu \mathrm{L}$. The primer 4 (5'-AAGAGCCCGT-3') was selected for $C$. albicans isolates after testing six primer sequences for exclusive use in RAPD (GE Healthcare, United Kingdom). Amplification was performed in an automated thermal cycler (Progene, USA) and consisted of one initial denaturation cycle at $95^{\circ} \mathrm{C}$ for 5 min, 45 denaturation cycles at $95{ }^{\circ} \mathrm{C}$ for $1 \mathrm{~min}$, annealing at $32{ }^{\circ} \mathrm{C}$ for $1 \mathrm{~min}$, extension at $72{ }^{\circ} \mathrm{C}$ for 2 min, and a final extension cycle for $10 \mathrm{~min}$. DNA samples were tested in duplicate. As negative control, ultrapure water was used. PCR products were electrophoresed on polyacrylamide gels in TBE buffer pH 8 and stained with ethidium bromide. Fragment sizes were determined by comparison with a 100-bp DNA ladder (Invitrogen Ltda, Brazil) and visualized by UV transillumination Mini Bis Pro (Bio-Imaging Systems, Ltd, Israel). RAPD profiles of C. albicans clinical isolates were compared to that of the type $C$. albicans (ATCC) 76615 strain. All experiments were carried out in duplicate to assess reproducibility.

\subsection{Analysis of Data and Dendrogram Generation}

RAPD profiles were analyzed by Bionumerics version 5.10 (Applied Maths, Kortrijk, Belgium). The similarity coefficient $\left(\mathrm{S}_{\mathrm{AB}}\right)$ between patterns for every pair of isolates $\mathrm{A}$ and $\mathrm{B}$ was computed with the formula $\mathrm{S}_{\mathrm{AB}}=22_{\mathrm{E}} /\left(2_{\mathrm{E}}+a+b\right)$, where $E$ is the number of common bands in the patterns $\mathrm{A}$ and $\mathrm{B} ; a$ is the number of bands in pattern A with no correlates in pattern B; and $b$ is the number of bands in pattern $\mathrm{B}$ with no correlates in pattern A. Dendrograms based on $\mathrm{S}_{\mathrm{AB}}$ values were generated through dendrograms by UPGMA (unweighted pair-group method using arithmetic average) methods (1.5\% optimization and $2 \%$ tolerance) implemented in the Bionumerics software. An $\mathrm{S}_{\mathrm{AB}}$ value of 1.00 (100.0\%) indicated that the banding patterns for strain A were identical to those for strain $\mathrm{B} ; \mathrm{S}_{\mathrm{AB}}$ values of 0.80-0.99 (80.0-99.0\%) represented highly similar but not identical strains and $\mathrm{S}_{\mathrm{AB}}$ values below 0.80 indicated unrelated strains $[7,9]$.

\subsection{Antifungal Susceptibility Testing}

Standard antifungal susceptibility tests were carried out in $140 \mathrm{C}$. albicans isolates according to the reference microdilution method established by the EUCAST (European Committee on Antimicrobial Susceptibility Testing) [16]. AMB, KTC, FLC and ITC were the antifungal compounds employed (Sigma-Aldrich Brazil Ltda.). With regards to FLC susceptibility, isolates were classified as susceptible (S) for $\mathrm{MIC} \leq 8.0 \mathrm{mg} / \mathrm{L}$; intermediate (I) for MIC equal to $4.0 \mathrm{mg} / \mathrm{L}$; and resistant (R) for $\mathrm{MIC} \geq 8.0 \mathrm{mg} / \mathrm{L}$, according to EUCAST [16]. For KTC and ITC, isolates were classified as susceptible (S), SDD (susceptible dose-dependent) and resistant (R), according to the CLSI (Clinical and Laboratory Standards Institute) [17] in values of $\geq 1 \mathrm{mg} / \mathrm{L}, 0.25-0.5 \mathrm{mg} / \mathrm{L}$, and $\leq 0.125$ $\mathrm{mg} / \mathrm{L}$ for R, SDD and S, respectively. Since interpretative criteria for AMB susceptibility have not been established so far by either EUCAST or CLSI, 
isolates were considered susceptible with MICs $\leq 1$ $\mathrm{mg} / \mathrm{L}$ and resistant with MICs $\geq 2 \mathrm{mg} / \mathrm{L}$ [18]. As positive controls, Candida krusei ATCC 6258 and C. parapsilosis ATCC 22019 were used.

\subsection{Statistical Analyses}

These were done by the Wilcoxon, Friedmann and Kruskall-Wallis tests, followed by Dunn's test for multiple comparisons. Comparison of frequencies was carried out by Fisher's exact test. All tests were performed using the SAS (statistical analysis system), version 6.12 (Institute Inc. USA) (SAS). Significance was set at $p<0.05$.

\section{Results}

Only 6 out of 59 HIV-infected individuals were symptomatic of oral candidiasis in two or more visits during the period under study. In all patients, Candida spp. were isolated in variable frequencies, that is, in 41 patients $(69.50 \%)$ in two visits, 13 patients $(22.0 \%)$ in three visits, 4 patients $(6.8 \%)$ in four visits, and only one patient (1.7\%) in all five visits, for a final sampling of 142 oral swabs. These were plated on CHROMagar; in them, 153 isolates were identified, 140 of them $C$. albicans and 13 non-Candida albicans. From one oral swab two colonies with different green coloration were isolated (Table 2). Isolation of the same species in sequential oral swabs was observed in 52 out of 59 patients $(88.1 \%)$, C. albicans in $50(84.7 \%), C$. albicans together with C. parapsilosis in the other two (3.4\%). In one case only Candida non-albicans were isolated in sequential oral swab (C. glabrata in the first and C. tropicalis in the second visit). In the remaining six cases, different species or species associations were observed in sequential visits, totaling 15 C. albicans, three C. tropicalis, two C. glabrata, one $C$. parapsilosis and one C. krusei isolates.

The identification of 140 C. albicans isolates was confirmed by PCR using amplification of the primers CAL5/NL4CAL and CDU2/NL4CAL (Fig. 1).

\subsection{RAPD Genotyping and Dendrogram Analysis of Uential Clinical C. albicans Isolates}

The visual and gross examination of the fingerprint profiles of sequential C. albicans isolates indicated 61 different molecular profiles (Fig. 2). They were grouped into twenty main clusters, with $77.9 \%$ similarity (Fig. 3). $\mathrm{S}_{\mathrm{AB}}$ ranged from 0.46 to 1.0 with mean plus standard deviation of $0.779 \pm 0.178$. Clusters (I to XX) were derived at a threshold $\mathrm{S}_{\mathrm{AB}}$ of $80.0 \%$.

Seventeen samples isolated from six individuals with symptomatic oral candidiasis were grouped into seven clusters with $78.0 \%$ similarity among them; $\mathrm{S}_{\mathrm{AB}}$ ranged from 0.54 to 1.0 , with mean plus standard deviation of $0.78 \pm 0.174$ (Fig. 4).

Four of the seven clusters generated two to six subclusters with $\mathrm{S}_{\mathrm{AB}}$ varying from 0.80 to 0.90 (Table 3). Clusters I and II included two strains with $\mathrm{S}_{\mathrm{AB}} 0.70$ between them, while cluster $\mathrm{V}$ included one strain with $\mathrm{S}_{\mathrm{AB}} 0.76$ to clusters III and IV, suggesting unrelated isolates. On the contrary, cluster VII grouped two isolates with $\mathrm{S}_{\mathrm{AB}} 0.90$, suggesting highly related strains (Table 3).

Table 2 Distribution of Candida spp. isolated from 59 HIV-infected individuals as the number of oral swabs per patient and phenotypic identification.

\begin{tabular}{llllll}
\hline Oral swabs (no./patient) & Patients (no.) & Oral swabs (total) & Isolates (no.) & \multicolumn{2}{c}{ Phenotypic identification } \\
\cline { 5 - 6 } & & & & C. albicans (no.) & Non-Candida albicans (no.) \\
\hline 2 & 41 & 82 & 88 & 79 & 09 \\
4 & 13 & 39 & 43 & $40^{\text {a }}$ & 03 \\
5 & 04 & 16 & 16 & 16 & 00 \\
Total & 01 & 05 & 06 & 05 & 01 \\
\hline
\end{tabular}

${ }^{\mathrm{a}}$ Two C. albicans isolates from the third oral swab of a patients, with tonality mildly different. 
Genotyping and Antifungal Susceptibility Profile of Sequential Candida albicans Isolated from the Oral Cavity of HIV-Infected Individuals

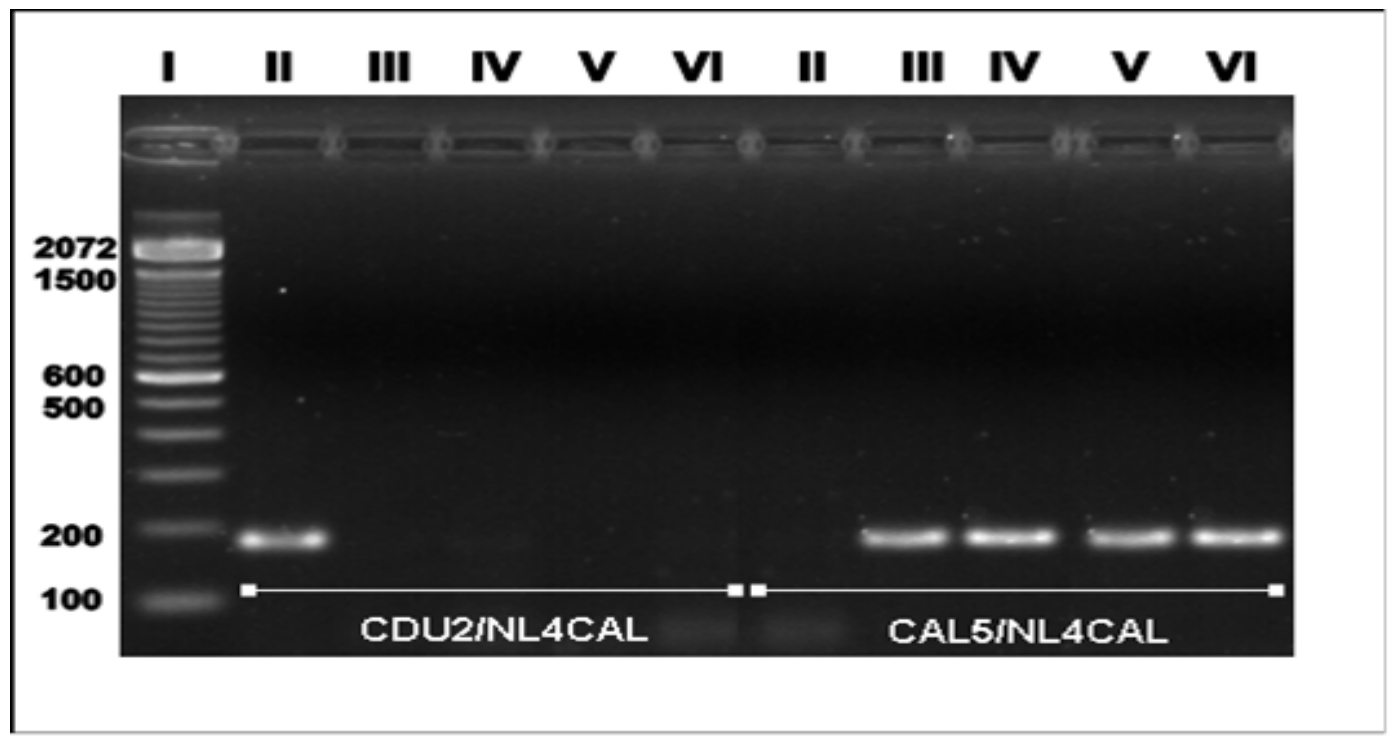

Fig. 1 Molecular differentiation between Candida albicans and Candida dubliniensis by amplification primer CAL5/NL4CAL and CDU2/NL4CAL by the PCR, product of 175 bp. Line I, 100-bp ladder; line II, C. dubliniensis (CBS 9768); line III, C. albicans (ATCC 76615); lanes IV to VI Candida albicans isolates from the oral cavity of human immunodeficiency virus infected individuals.

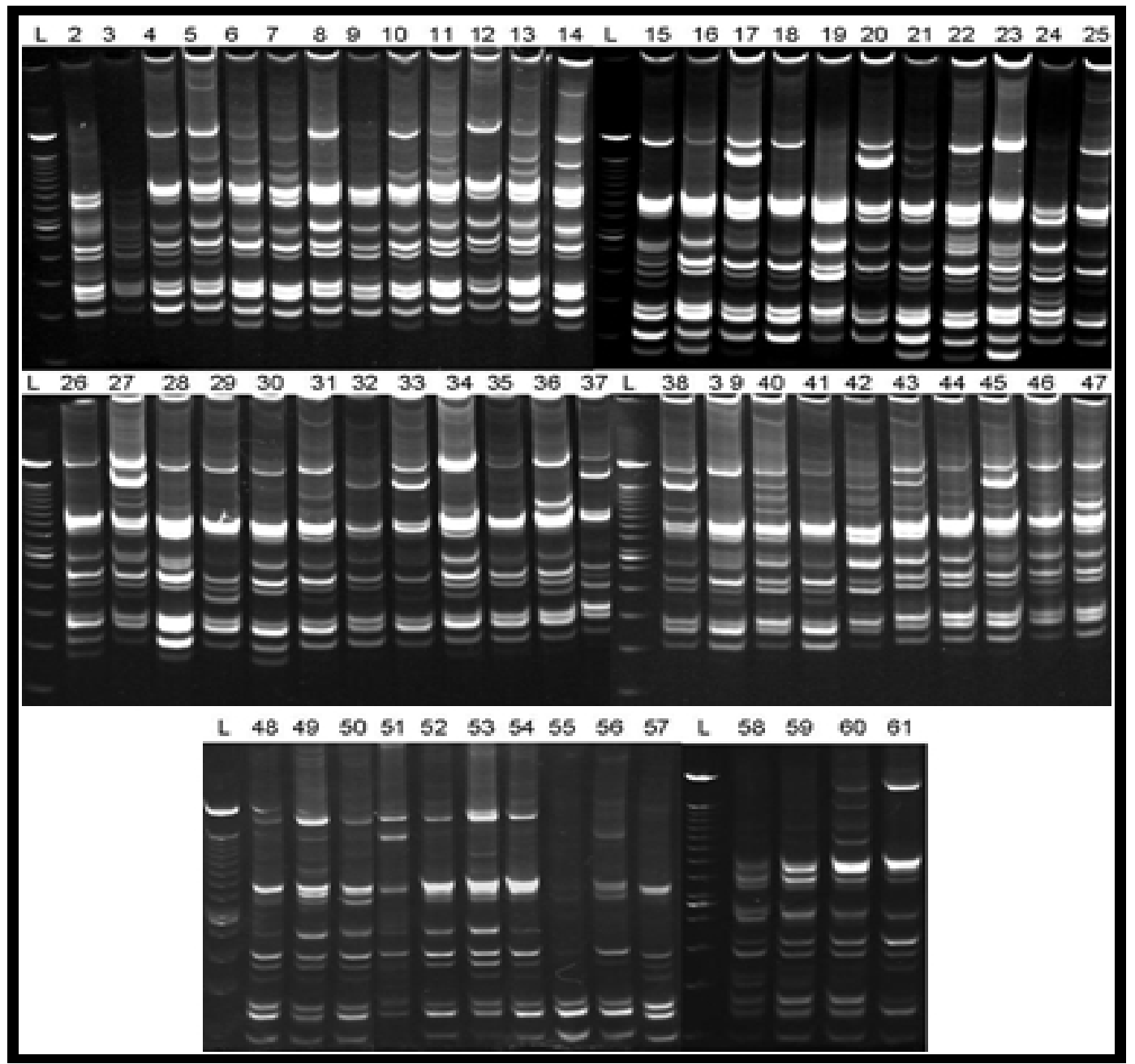

Fig. 2 RAPD profiles of Candida albicans obtained from 140 isolates of human immunodeficiency virus individuals. 

Isolated from the Oral Cavity of HIV-Infected Individuals

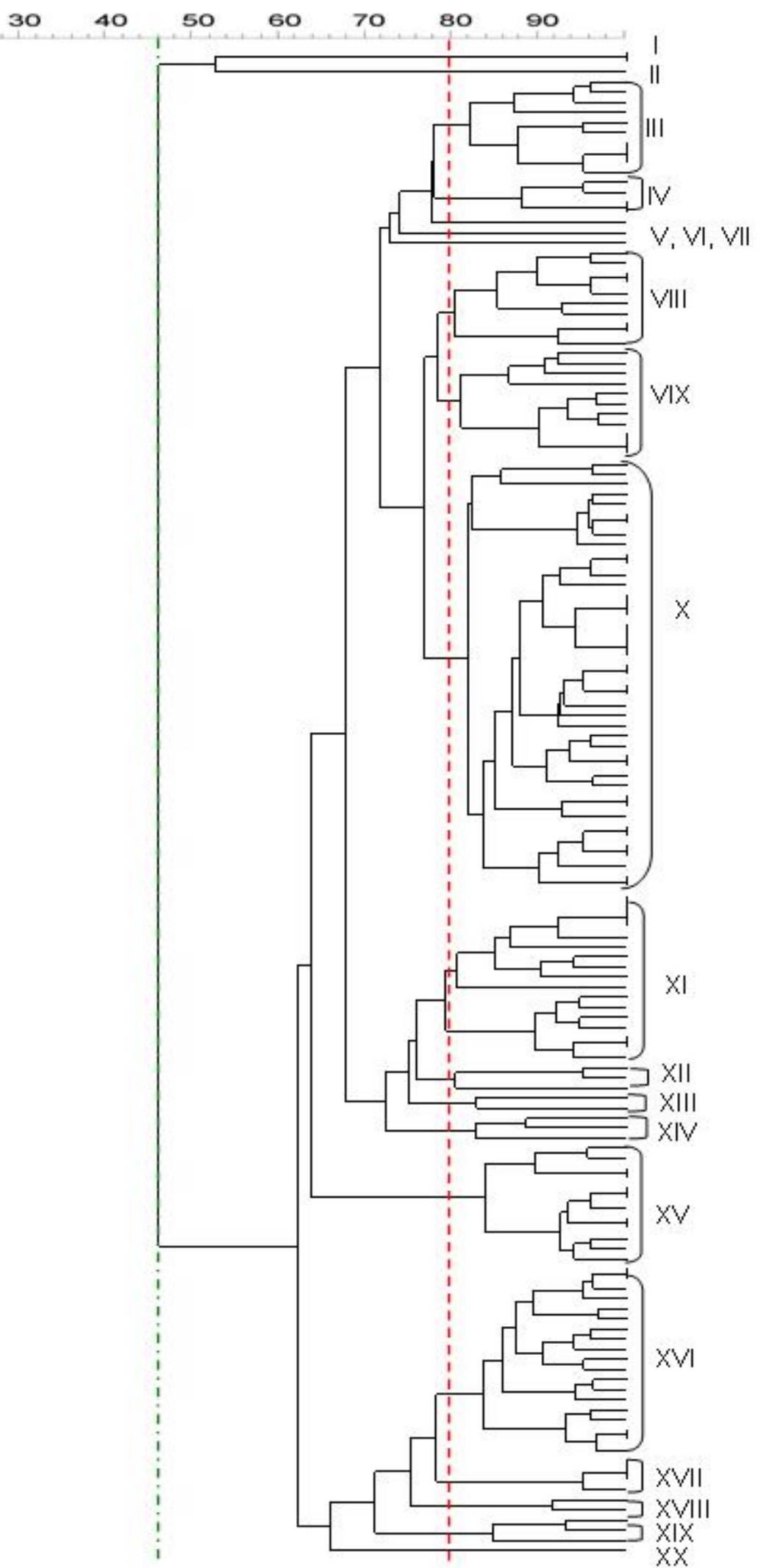

Fig. 3 Dendrograms generated for sequential Candida albicans from human immunodeficiency virus infected individuals. Vertical dashed line mark the positions of $S_{A B}$ values of 0.46 and 0.80 , clustering. Heavy vertical lines to the right of each dendrogram mark the position of clusters. 

Isolated from the Oral Cavity of HIV-Infected Individuals

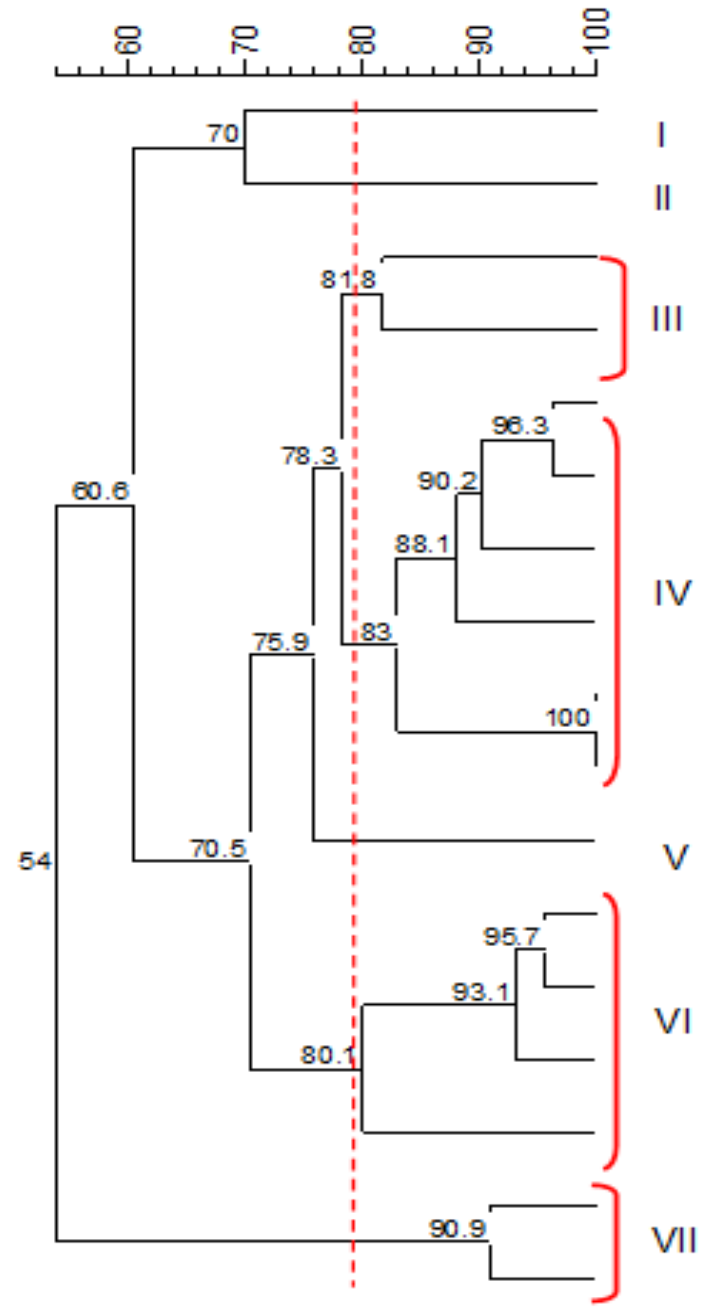

Fig. 4 Dendrograms generated by seventeen oral Candida albicans isolates from six human immunodeficiency virus patients with symptomatic oral candidiasis. Vertical dashed line mark the position of SAB 0.80 , clustering. Heavy vertical lines to the right of each dendrogram mark the position of clusters.

Table 3 Distribution oral Candida albicans isolates obtained by sequential evaluations of 40 human HIV individuals colonized by Candida albicans, and six AIDS-patients with oral candidiasis according to clusters and coefficient of similarity $\left(\mathrm{S}_{\mathrm{AB}}\right)$ of the subclusters.

\begin{tabular}{|c|c|c|c|c|c|}
\hline \multicolumn{3}{|c|}{$\begin{array}{l}\text { Oral-colonization } \\
\text { (41 HIV-infected individuals) }\end{array}$} & \multicolumn{3}{|c|}{$\begin{array}{l}\text { Oral candidiasis } \\
\text { (06 AIDS-patients) }\end{array}$} \\
\hline $\begin{array}{l}\text { Cluster } \\
\text { number }\end{array}$ & $\begin{array}{l}\text { Strains } \\
\text { (no.) }\end{array}$ & $\mathrm{S}_{\mathrm{AB}}$ & $\begin{array}{l}\text { Cluster } \\
\text { number }\end{array}$ & $\begin{array}{l}\text { Strains } \\
\text { (no.) }\end{array}$ & $\mathrm{S}_{\mathrm{AB}}$ \\
\hline I & 11 & 0.84 & I & 1 & - \\
\hline II & 5 & 0.84 & II & 1 & - \\
\hline III & 6 & 0.81 & III & 2 & 0.81 \\
\hline IV & 1 & - & IV & 6 & 0.83 \\
\hline V & 4 & 0.88 & $\mathrm{~V}$ & 1 & - \\
\hline VI & 3 & 0.96 & VI & 4 & 0.80 \\
\hline VII & 3 & 0.85 & VII & 2 & 0.90 \\
\hline VIII & 9 & 0.87 & & & \\
\hline IX & 2 & $1.0^{\mathrm{a}}$ & & & \\
\hline Total & 44 & - & Total & 17 & - \\
\hline
\end{tabular}

${ }^{\mathrm{a}}$ Identical strains. 
A similar dendrogram, based on the $\mathrm{S}_{\mathrm{AB}}$ values of genetically related isolates, was generated for the other 44 samples from individuals without clinical manifestations of candidiasis. Due to the large number of strains, their RAPD profiles were carried out only in samples with different appearance by visual analysis (Fig. 2). The Dendron database formed nine clusters with $87.0 \%$ similarity among them and $\mathrm{S}_{\mathrm{AB}}$ ranging from 0.46 to 1.0, with mean plus standard deviation of $0.87 \pm 0.123$. Eight of the nine clusters showed two to 11 subclusters with $\mathrm{S}_{\mathrm{AB}}$ varying from 0.81 to 1.0 (Table 3). Cluster II contained two identical strains ( $\mathrm{S}_{\mathrm{AB}}$ equal 1.0), and three other genetically related strains, with $\mathrm{S}_{\mathrm{AB}}$ 0.93. Two identical strains were found in cluster III, together with four other strains connecting at an $\mathrm{S}_{\mathrm{AB}} \mathbf{0} \cdot 81$. Cluster IV was composed of only one isolate, with $\mathrm{S}_{\mathrm{AB}} 0.70$ to cluster III. Cluster VIII had nine isolates with $\mathrm{S}_{\mathrm{AB}}$ 0.87, and isolates connected to each other with high similarity $\left(\mathrm{S}_{\mathrm{AB}}>\right.$ 0.93); finally, cluster IX with $S_{A B} 0.10$ showed a $\mathrm{S}_{\mathrm{AB}} 0.46$ to all other clusters, suggesting unrelated isolates.

3.2 Comparison of the Similarity in Sequential Evaluation of C. albicans Isolates as to Host-Parasite Relationship

The degree of similarity among C. albicans was higher $(p=0.048)$ for isolates from colonization $\left[\mathrm{S}_{\mathrm{AB}}<\right.$ 0.80 for 13 (25.0\%) and $S_{A B} \geq 0.80$ for $29(75.0 \%)$ patients] than those from infection $\left[\mathrm{S}_{\mathrm{AB}}<0.80\right.$ for four (67.0\%) and $\mathrm{S}_{\mathrm{AB}} \geq 0.80$ for two (33.0\%) patients].

Antifungal susceptibility testing, all tested isolates grew after $24 \mathrm{~h}$ incubation and were susceptible to the different antifungal agents evaluated. Median and ranges of the MICs were 0.25 [0.125-1.0] for FLC, 0.015 [0.015-0.06] for ITC, 0.015 [0.015-0.06] for KTC, and 0.25 [0.03-1.0] for AMB. In addition, no differences in MICs of any given antifungal compound were calculated for sequential C. albicans isolates ( $p>$ 0.05).

\section{Discussion}

The pathogenicity of Candida species is a result of the characteristics of the strains, immunological status of the host, and conditions of infection sites. Oropharyngeal candidiasis (OPC) has been considered the most common opportunistic infection among HIV-patients [19]. Due to recurrent episodes of candidiasis, patients are frequently subjected to repeated courses of FLC therapy [5, 6, 13, 20]. After the introduction of HAART (highly active antiretroviral therapy), the occurrence of OPC has receded [19, 21, 22]. However, OPC is frequent among patients with low compliance or poor response to HAART [22]. Longitudinal studies on the oral colonization and infection patterns of the human pathogen C. albicans in HIV-infected individuals are scarce. Using RFLP as the method of analysis, Barchiesi et al. [23], carried out a sequential evaluation of five AIDS-patients with recurrent candidiasis during a 5-year period and observed that four of them maintained the similarity of isolates along time. Samaranayake et al. [9] selected five C. albicans colonies from each sample obtained in the visits, during a 1-year, totalizing 443 isolates from AIDS-patients, 10 of them with symptomatic candidiasis in one or more visits, and analyzed them by RAPD. All patients presented C. albicans isolates with high degree of similarity $\left(\mathrm{S}_{\mathrm{AB}} \geq 0.80\right)$ to isolates from other visits. From 11 of the 16 patients, genetically identical isolates were taken in two to four sequential visits, three of which colonized and eight infected. These authors observed a higher degree of similarity among OPC patients. Several isolates presented a similarity degree between $\mathrm{S}_{\mathrm{AB}} \quad 0.70$ and 0.80 , suggesting a genetic shuffling during HIV disease progression. Similar genetic shuffling in C. albicans, leading to evolution of subtypes during HIV disease progression, has been reported by other authors [24-29]. Our study showed higher prevalence of similarity for colonizing (75.0\%) than infecting (33.3\%) C. albicans 

Isolated from the Oral Cavity of HIV-Infected Individuals

isolates from HIV-positive individuals, with degrees of similarity of $\mathrm{S}_{\mathrm{AB}} 0.87$ and 0.78 , respectively. In our study, we compared isolates above and under $\mathrm{S}_{\mathrm{AB}} 0.80$ of similarity, from $59 \mathrm{HIV}$-infected patients, a higher number than those reported by Barchiesi et al. (1997) and Samaranayake, et al. (2003) [9, 23].

To switch from saprophytic (colonization) to pathogenic (infection) state, Candida needs to increase both its adhesive properties to attach to host components and its production of lytic enzymes to penetrate the tissues $[8,13]$. The main hydrolytic enzyme involved in its virulence is the Sap (secretory aspartyl proteinase), belonging to the same protease class as HIV protease, the target for the PIs (proteinase inhibitors) used in AIDS therapy. In contrast to the very small and structurally simplified HIV proteinase, Saps are larger and more complex. Thus, the inhibition of these proteinases by PIs would reduce the virulence of C. albicans, as demonstrated when the inhibitory activities of PIs were comparable to those exerted by pepstatin A, a prototypal inhibitor of Saps [30].

When colonization occurs, Candida species may be balanced and more stable, a behavior that changes to unbalanced in a pathogenic state, under the pressure of several factors, among them, intense production of no less than nine Saps (SAP1 to SAP9) encoded by a single gene family [30, 31], the cell-mediated immune response of the host, and the antifungal and antiretroviral substances in use for therapeutical reasons. This may explain the higher similarity among colonizing than infecting C. albicans isolates, found in our experiments, as it was observed by the failure of oral host defenses in AIDS patients, indicated by the increase in Candida spp. Carriage [3, 22] may simply provide highly adapted strains to the oral cavity with an opportunity to compete with and subsequently replace the original oral commensal strains, thus causing OPC [29] as a consequence, Candida species may be unbalanced and less stable. Our study indicates that the sequential evaluation of Candida species produced a few Candida non-albicans species, a result not shared by Barchiesi et al. and Samaranayake, et al., who did not report the isolation of such species [9, 23].

The finding of resistant $C$. albicans strains from AIDS- patients with OPC suggests that fungal strains may become unresponsive to certain drug treatments [2]. Thus, determining the sensitivity of sequential $C$. albicans strains isolates from HIV-positive individuals becomes imperative to establish an effective drug therapy. Our results demonstrated that Candida isolates did not change their pattern of susceptibility of any of the antifungals used throughout the 5 years period of the study. The fact that only $12.7 \%$ patients had been exposed to previous antifungal treatments, could explain our results. These findings are very important from a clinical point of view, constituting a safe guide for therapy. While Barchiesi et al. (1997) and Samaranayake et al. (2003) [9, 23] did not evaluate the antifungal susceptibility of sequentially isolated $C$. albicans strains, Korting et al. (1999) evaluated the antifungal susceptibilities and the biotype profiles of $C$. albicans isolates from 62 AIDS-patients. They found a predominant biotype among these isolates and a new biotype in $27.3 \%$ of patients. Antifungal susceptibility testing revealed higher MICs for amphotericin B, flucytosine, ketoconazole, and itraconazole for $C$. albicans isolates from patients with advanced AIDS stages and prolonged exposure to antifungal agents [30]. These results disagree with our findings and suggested that long-term antifungal therapy for OPC in AIDS-patients may result in decreased susceptibility to commonly used antifungal. In contrast to the studies of Pfaller et al. (1994), we found evidence of low strain diversity among C. albicans isolates from AIDS-patients with OPC; $38 \%$ of their patients were infected or colonized with the same DNA subtype on sequential cultures, and $62 \%$ of the patients experienced episodes of infection with two or more different C. albicans DNA subtypes. Differences in patient populations, frequency of cultures, and duration of follow-up, intensity of exposure to specific antifungal agents and methods of DNA subtyping may 
all have contributed to the different conclusions of the studies described above Ref. [26].

The evidence that $C$. albicans reproduces in a clonal way, Pujol et al. (1993) and Tibayrenc, (1997) suggests that it can adapt to its environments if such niches are stable [27, 28]. Alternatively, when exposed to frequent environmental changes, these microorganisms respond through micro-evolutionary selection based on their capacity to adapt to varied niches [28]. Oral mucous membranes of HIV-infected individuals with reduced cell-mediated immune response, associated with the pressure of antifungal and antiretroviral compounds, can behave as a dynamic environment subjected to frequent changes.

\section{Conclusions}

Our findings showed that in our region C. albicans remains the major species colonizing or infecting the oral cavity of HIV-positive individuals, with a high degree of similarity, mainly among the colonized individuals; all isolates were susceptible to AMB, KTC, FLC and ITC and maintained its susceptibility along the studied period.

\section{Conflicts of Interest}

None.

\section{References}

[1] Richardson, J. P., and Moyes, D. L. 2015. "Adaptive Immune Responses to Candida albicans Infection.” Virulence 6 (4): 327-37.

[2] Anwar-Khan, P., Malik, A., and Subhan Khan, H. 2012. "Profile of Candidiasis In HIV Infected Patients.” Iranian Journal of Microbiology 4 (4): 204-9.

[3] Junqueira, J. C., Vilela, S. F. G., Rossoni, R. D., Barbosa, J. O., Costa, A. C. B. P., Rasteiro, V. M. C., and Jorge, A. O. C. 2012. "Oral Colonization by Yeasts in HIV-Positive Patients in Brazil.” Rev. Inst. Med. Trop Sao Paulo 54 (1): 17-24. http://doi.org/10.1590/S0036-46652012000100004.

[4] Maheshwari, M., Kaur, R., and Chadha, S. 2016. “Candida Species Prevalence Profile in HIV Seropositive Patients from a Major Tertiary Care Hospital in New Delhi, India.” Journal of Pathogens, 8. http://doi.org/10.1155/2016/6204804.
[5] Whaley, S. G., Berkow, E. L., Rybak, J. M., Nishimoto, A. T., Barker, K. S., Rogers, P. D., and Rogers, P. D. 2017. "Azole Antifungal Resistance in Candida albicans and Emerging Non-albicans Candida Species.” Frontiers in $\begin{array}{llll}\text { Microbiology } & 7 & \text { (1): } & 1-12 .\end{array}$ http://doi.org/10.3389/fmicb.2016.02173.

[6] Johnson, E. M., Warnock, D. W., Luker, J., Porter, S. R., and Scully, C. 1995. "Emergence of Azole Drug Resistance in Candida Species from HIV-Infected Patients Receiving Prolonged Fluconazole Therapy for Oral Candidosis.” Journal of Antimicrobial Chemotherapy 35 (1): 103-14.

[7] Soll, D. R., Staebell, M., Langtimm, C., Pfaller, M., Hicks, J., and Rao, T. V. G. 1988. "Multiple Candida Strains in the Course of a Single Systemic Infection.” Journal of Clinical Microbiology 26 (8): 1448-59.

[8] Bai, F. 2014. "Association of Genotypes with Infection Types and Antifungal Susceptibilities in Candida albicans as Revealed by Recent Molecular Typing Strategies.” Mycology $\quad 5 \quad$ (1): $1-9$. http://doi.org/10.1080/21501203.2014.899525.

[9] Samaranayake, Y. H., Samaranayake, L. P., Dassanayake, R. S., Yau, J. Y. Y., Tsang, W. K., Cheung, B. P. K., and Yeung, K. W. S. 2003. "Genotypic Shuffling of Sequential Clones of Candida albicans in HIV-Infected Individuals with and without Symptomatic Oral Candidiasis.” Journal of Medical Microbiology 52: 349-59. http://doi.org/10.1099/jmm.0.04972-0.

[10] Vargas, K., Messer, S. A., Pfaller, M., Lockhart, S. R., Stapleton, J. T., Hellstein, J., and Soll, D. R. 2000. "Elevated Phenotypic Switching and Drug Resistance of Candida albicans from Human Immunodeficiency Virus-Positive Individuals Prior to First Thrush Episode.” Journal of Clinical Microbiology 38 (10): 3595-607.

[11] Xu, J., Vilgalys, R., and Mitchell, T. G. 1999. "Lack of Genetic Differentiation between Two Geographically Diverse Samples of Candida albicans Isolated from Patients Infected with Human Immunodeficiency Virus.” Journal of Bacteriology 181 (4): 1369-73.

[12] Lyon, P. J., Moraes, K. C. M., Moreira, L. M., Aimbire, F., and Resende, M. A. 2010. "Candida albicans: Genotyping Methods and Clade Related Phenotypic Characteristics.” Brazilian Journal of Microbiology 41: 841-9.

[13] Niimi, M., Firth, N. A., and Cannon, R. D. 2010. “Antifungal Drug Resistance of Oral Fungi.” Odontology 98 (1): 15-25.

[14] Green, M. R., and Sambrook, J. 2012. Molecular Cloning: a Laboratory Manual. (J. Sambrook \& D. W. Russell, Eds.) (4th ed., Vol. 1). New York: Cold Spring Harbor Laboratory Press.

[15] Mannarelli, B. M., and Kurtzman, C. P. 1998. "Rapid Identification of Candida albicans and Other Human 


\section{Genotyping and Antifungal Susceptibility Profile of Sequential Candida albicans Isolated from the Oral Cavity of HIV-Infected Individuals}

Pathogenic Yeasts by Using Short Oligonucleotides in a PCR.” Clin Microbiol. 36 (6): 1634-41.

[16] EUCAST. 2015. "EUCAST Definitive Document E. DEF 7.3 Method for the Determination of Broth Dilution Minimum Inhibitory Concentrations of Antifungal Agents for Yeasts.” EUCAST, 1-21.

[17] CLSI. 2012. "Reference Method for Broth Dilution Antifungal Susceptibility Testing of Yeasts.” Approved standard. In CLSI (Ed.), CLSI Document M27-S4 (4th ed., p. 25). Wayne, PA: Clinical and Laboratory Standards Institute.

[18] Nguyen, M. H., Clancy, C. j, Yu, V. L., Yu, Y. C., Morris, A. J., Snydman, D. R., Rinaldi, M. G., et al. 1998. "Do in vitro Susceptibility Data Predict the Microbiologic Response to Amphotericin B? Results of a Prospective Study of Patients with Candida Fungemia.” J. Infect. Dis. 177 (2): 425-30.

[19] Kaur, R., Dhakad, M. S., Goyal, R., Bhalla, P., and Dewan, R. 2016. "Spectrum of Opportunistic Fungal Infections in HIV/AIDS Patients in Tertiary Care Hospital in India." Canadian Journal of Infectious Diseases and Medical Microbiology, 7.

[20] Korting, H. C., and Schöllmann, C. 2008. "The Significance of Itraconazole for Treatment of Fungal Infections of Skin, Nails and Mucous Membranes." Journal of the German Society of Dermatology 7 (1): 11-9.

[21] Greenspan, D., Gange, S. J., Phelan, J. A., Navazesh, M., Alves, M. E. A. F., MacPhail, L. A., Greenspan, J. S., et al. 2004. "Incidence of Oral Lesions in HIV-1-Infected Women: Reduction with HAART." Journal of Dental Research 83 (2): 145-50.

[22] Moris, D. V, Melhem, M. S. C., Martins, M. A., and Menders, R. P. 2008. "Oral Candida spp. Colonization in Human Immunodeficiency Virus-Infected Individuals.” $J$. Venom Anim Toxins Incl. Trop Dis. 14 (2): 224-57.

[23] Barchiesi, F., Di Francesco, L. F., Compagnucci, P., Arzeni, D., Cirioni, O., and Scalise, G. 1997. "Genotypic Identification of Sequential Candida albicans Isolates from AIDS Patients by Polymerase Chain Reaction Techniques.” European Journal of Clinical Microbiology and Infectious Diseases 16 (8): 601-5.

[24] Ball, L. M., Bes, M. A., Theelen, B., Boekhout, T., Egeler, R. M., Kuijper, E. J., and Icrobiol, J. C. L. I. N. M. 2004. "Significance of Amplified Fragment Length Polymorphism in Identification and Epidemiological
Examination of Candida Species Colonization in Children Undergoing Allogeneic Stem Cell Transplantation." Journal of Clinical Microbiology 42 (4): 1673-9. http://doi.org/10.1128/JCM.42.4.1673.

[25] Metzgar, D., Field, D., Haubrich, R., and Wills, C. 1998. "Random Amplification of Polymorphic DNA and Microsatellite Genotyping of Pre- and Post-treatment Isolates of Candida spp . from Human Immunodeficiency Virus-Infected Patients on Different Fluconazole Regimens.” Journal of Clinical Microbiology 36 (8): 2308-13.

[26] Pfaller, M. A., Rhine-chalberg, J., Redding, S. W., Smith, J., Farinacci, G., Fothergill, A. W., and Rinaldi, M. G. 1994. "Variations in Fluconazole Susceptibility and Electrophoretic Karyotype among Oral Isolates of Candida albicans from Patients with AIDS and Oral Candidiasis.” Journal of Clinical Microbiology 32 (1): 59-64.

[27] Pujol, C., Reynest, J., Raymond, M., Tibayrenc, M., Ayalaii, F. J., Janbont, O. I. S., Bastide, J., et al. 1993. "The Yeast Candida albicans Has a Clonal Mode of Reproduction in a Population of Infected Human Immunodeficiency Virus-Positive Patients.” Proc. Natl Acad. Sci. 90 (8): 9456-9.

[28] Tibayrenc, M. 1997. "Are Candida albicans Natural Populations Subdivided?” Trends in Microbiology 5 (7): 253-4.

[29] Schmid, J. A. N., Odds, F. C., Wiselka, M. J., Nicholson, K. G., and Soll, D. R. 1992. "Genetic Similarity and Maintenance of Candida albicans Strains from a Group of AIDS Patients, Demonstrated by DNA Fingerprinting." Journal of Clinical Microbiology 30 (4): 935-41.

[30] Korting, H. C., Schaller, M., Eder, G., Hamm, G., and Hube, B. 1999. "Effects of the Human Immunodeficiency Virus (HIV) Proteinase Inhibitors Saquinavir and Indinavir on in vitro Activities of Secreted Aspartyl Proteinases of Candida albicans Isolates from HIV-Infected Patients." Antimicrobial Agents and Chemotherapy 43 (8): 2038-42.

[31] Chin, V. K., Lee, T. Y., Rusliza, B., and Chong, P. P. 2016. "Dissecting Candida albicans Infection from the Perspective of $C$. albicans Virulence and Omics Approaches on Host-Pathogen Interaction: A Review." International Journal of Molecular Sciences 17: 1643. http://doi.org/10.3390/ijms17101643. 